\title{
Inference for generalized exponential distribution based on generalized order statistics
}

\author{
Eldesoky E. Afify ${ }^{1,2} *$ \\ ${ }^{l}$ Department of Basic Engineering Sciences, Faculty of Engineering, Menoufiya University, Shibeen El-Kom, Egypt \\ ${ }^{2}$ Department of Mathematics, College of Sciences and Arts, Al Maznab, Al Qassim University, KSA \\ *Corresponding author E-mail: eldesoky@yahoo.com
}

Copyright $\odot 2015$ Eldesoky E. Afify. This is an open access article distributed under the Creative Commons Attribution License, which permits unrestricted use, distribution, and reproduction in any medium, provided the original work is properly cited.

\begin{abstract}
Estimation of a parameter of generalized exponential distribution (gexp) is obtained based on generalized order statistics. The maximum likelihood and Bayes methods are used for this purpose. Survival function and hazard rate are also computed. Estimation based on upper record values from generalized exponential distribution is obtained as a special case and compared by simulated data.
\end{abstract}

Keywords: Generalized Exponential Distribution; Maximum Likelihood; Bayes Estimation; Record Values; Survival Function; and Hazard Rate Function.

\section{Introduction}

The concept of generalized order statistics (gOS) was introduced in [1]. A variety of order models of random variables is contained in this concept. [2], [3] discussed gOS from exponential and power function distributions. [4] Obtained some recurrence relations of moments for order statistics from generalized exponential distribution (Gexp).

The minimum variance linear unbiased estimators, the best linear invariant estimators and maximum likelihood estimators based on n-selected generalized order statistics are presented for the parameters of the Burr XII distribution in [5].

The maximum likelihood estimation, Bayesian estimation using Jeffrey prior and the extension of Jeffrey prior information for estimating the parameters of the Weibull distribution of the life time is presented see [6]. The maximum likelihood and the Bayesian methods are applied to estimate the unknown parameters of the Weibull extension model by using the generalized order statistics; also asymptotic variance-covariance matrix of the estimators is given in [7]. [8] Estimated the reliability based on Pareto distribution. [9] Estimated the parameters of an exponentiated inverted Weibull distribution by Bayesian and non-Bayesian methods of estimation, she compared them through confidance intervals, and their mean squared error values. [10] derived the maximum likelihood estimators of an accelerated life tests model, prediction of scale parameter under usual conditions is obtained and reliability function at a certain mission time under the same conditions is predicted. The maximum likelihood estimation for the unknown parameters of the generalized exponential distribution, they suggested Tierney- Kanane's approximation to obtain the bayes estimates saw [11]. In this paper, we use the maximum likelihood and Bayes methods for estimating the parameter of the generalized exponential distribution based on generalized order statistics. Record values are considered as a special case of gOS, survival function and hazard rate are also computed, mean absolute error and mean square errors are used to compare ML and Bayes estimation methods using simulated data generated from Gexp. By Maple V.

The pdf and cdf of generalized exponential distribution proposed are given respectively by

$$
\begin{array}{ll}
g(x)=(1-\alpha x)^{(1 / \alpha)-1}, & 0 \leq x \leq 1 / \alpha, 0 \leq \alpha<1 . \\
F(x)=1-(1-\alpha x)^{(1 / \alpha)}, & 0 \leq x \leq 1 / \alpha, \quad 0 \leq \alpha<1 .
\end{array}
$$


The survival function of the Gexp takes the following form

$$
S(x)=(1-\alpha x)^{\frac{1}{\alpha}}, \quad 0 \leq x \leq 1 / \alpha, \quad 0 \leq \alpha<1 .
$$

The hazard function of the Gexp is given by

$$
h(x)=\frac{1}{1-\alpha x}, \quad 0 \leq x \leq 1 / \alpha, \quad 0 \leq \alpha<1 .
$$

Suppose $X(1, n, m, k), X(2, n, m, k), \ldots, X(n, n, m, k), k \geq 1$ and $m$ being a real number, are $n$ generalized order statistics based on an absolutely continuous cumulative distribution function (cdf) $F(x)$ with probability density function (pdf) $\mathrm{f}(\mathrm{x})$, then they having joint density function of the form given in [12] as

$$
\begin{aligned}
& f_{X(1, n, m, k) \ldots, X(n, n, m, k)}\left(x_{1}, \ldots, x_{n}\right)=k\left(\prod_{j=1}^{n-1} \gamma_{j}\right)\left(\prod_{i=1}^{n-1}\left[1-F\left(x_{i}\right)\right]^{m} f\left(x_{i}\right)\right) \\
& {\left[1-F\left(x_{n}\right)\right]^{\gamma_{r}-1} f\left(x_{n}\right)}
\end{aligned}
$$

The pdf of $X_{(r, n, m, k)}(1 \leq r \leq n)$, is given as follows

$$
f_{X(r, n, m, k)}(x)=\frac{C_{r-1}}{(r-1) !}[1-F(x)]^{\gamma_{r}-1} f(x) g_{m}^{r-1}[F(x)]
$$

The joint pdf of $X_{(r, n, m, k)}$ and $X_{(s, n, m, k)}(1 \leq \mathrm{r}<\mathrm{s} \leq \mathrm{n})$ is given by

$$
\begin{aligned}
& f_{X(r, n, m, k), X(s, n, m, k)}(x, y)=\frac{C_{s-1}}{(r-1) !(s-r-1) !}[1-F(x)]^{m} g_{m}^{r-1}[F(x)] \\
& {\left[h_{m}(F(y))-h_{m}(F(x))\right]^{s-r-1}[1-F(y)]^{\gamma_{s}-1} f(x) f(y),}
\end{aligned}
$$

For $0<\mathrm{x}<1, \mathrm{x}<\mathrm{y}$ and $\mathrm{r}<\mathrm{s}$, where

$$
\begin{aligned}
& h_{m}(x)= \begin{cases}\frac{-1}{m+1}(1-x)^{m+1}, & m \neq-1 \\
-\log (1-x), & m=-1,\end{cases} \\
& \text { And } g_{m}(x)=\int_{0}^{x}(1-t)^{m} d t=h_{m}(x)-h_{m}(0), \gamma_{r}=k+(n-r)(m+1) \\
& C_{r-1}=\prod_{i=1}^{r} \gamma_{i}
\end{aligned}
$$

Note that for $\mathrm{m}=0, \mathrm{k}=1$ we obtain the joint pdf for ordinary order statistics, and when $\mathrm{m}=-1$, $\mathrm{k}=1$, we get the joint pdf of the upper record values.

\section{Maximum likelihood method}

From (1.1), (1.2) and (1.5) the maximum likelihood function is given by

$$
L\left(\alpha / X_{-}\right)=k\left(\prod_{j=1}^{n-1} \gamma_{j}\right) V\left(\alpha, x_{-}\right) \exp \left[\frac{H\left(\alpha, X_{-}\right)}{\alpha}\right],
$$

Where 
$V\left(\alpha, X_{-}\right)=\prod_{i=1}^{n}\left(\frac{1}{1-\alpha x_{i}}\right), H\left(\alpha, X_{-}\right)=(m+1) \sum_{i=1}^{n-1} \lg \left(1-\alpha x_{i}\right)+\gamma_{r} \lg \left(1-\alpha x_{n}\right)$

The maximum likelihood (ML) estimate of the parameter $\alpha$ can be obtained from

$\frac{\partial \log L}{\partial \alpha}=\left(\alpha^{2}-(m+1) \alpha\right) \sum_{i=1}^{n-1} w_{i}+\left(\alpha^{2}-\alpha \gamma_{r}\right) w_{n}-(m+1) \sum_{i=1}^{n-1} \log \left(1-\alpha x_{i}\right)-\gamma_{r} \log \left(1-\alpha x_{n}\right)=0$

Setting $m=0, k=1$ and $i=1,2, \ldots$ in (2.2), gives the likelihood equation for order statistics as

$\left(\alpha^{2}-\alpha\right) \sum_{i=1}^{n} w_{i}-\sum_{i=1}^{n} \log \left(1-\alpha x_{i}\right)+(n-r) \alpha w_{n}-(n-r) \log \left(1-\alpha x_{n}\right)=0$

Setting $\mathrm{m}=-1$ and $\mathrm{k}=1$ in (2.2), we have likelihood equation from record values

$\alpha^{2} \sum_{i=1}^{n} w_{i}-\alpha w_{n}-\lg \left(1-\alpha x_{n}\right)=0$

Where

$w_{i}=\frac{x_{i}}{1-\alpha x_{i}}, w_{n}=\frac{x_{n}}{1-\alpha x_{n}}$

Mle of $\alpha$ is obtained by solving (2.4) using Maple V.

\section{Bayes estimation}

The likelihood function (1.6) can be written as:

$L\left(\alpha / X_{-}\right)=k\left(\prod_{j=1}^{n-1} \gamma_{j}\right) \prod_{i=1}^{n}\left(1-\alpha x_{i}\right)^{-1} \exp \left[\frac{H(\alpha, X)}{\alpha}\right]$

Where $H\left(\alpha, X_{-}\right)=(m+1) \sum_{i=1}^{n-1} \lg \left(1-\alpha x_{i}\right)+\gamma_{r} \lg \left(1-\alpha x_{n}\right)$

We use the inverted gamma conjugate prior density for the parameter $\alpha$

$\pi(\alpha)=\frac{\beta^{p}}{\Gamma(p+1)} \alpha^{-p-1} e^{-\frac{\beta}{\alpha}}$

It follows from (3.1) and (3.2), that the posterior density of $\alpha$ is then

$$
\begin{aligned}
& f(\underset{-}{x})=\frac{\beta^{p}}{\Gamma(p+1)} \alpha^{-p-1} e^{-\frac{\beta}{\alpha}} k\left(\prod_{i=1}^{n-1} \gamma_{j}\right) \prod_{i=1}^{n-1}\left(1-\alpha x_{i}\right)^{-1} \exp \left[\frac{H(\alpha, x)}{\alpha}\right] \\
& H_{-}^{(X / \alpha)}=k\left(\prod_{i=1}^{n-1} \gamma_{j}\right) \frac{\beta^{p}}{\Gamma(P+1)} \prod_{i=1}^{n}\left(1-\alpha x_{i}\right)^{-1} \alpha^{-p-1} \exp \left[-\left(\frac{\beta-H(\alpha, X}{\alpha}\right)\right] \\
& P\left(X_{-}\right)=\int_{0}^{\infty} H\left(X_{-} / \alpha\right) d \alpha
\end{aligned}
$$

The posterior distribution of $\alpha$ is: 
$\Pi(\alpha / X)=\frac{H(X / \alpha)}{\int_{0}^{\infty} H(X / \alpha) d \alpha}=\frac{\alpha_{-}^{-p-1} e\left[-\left(\frac{\beta-H(\alpha, X)}{\alpha}\right)\right]}{\Gamma(p)\left[\beta+H\left(\alpha, X_{-}\right)\right]^{-p}}$

The bayes estimate of the parameter $\alpha$ is the mean of the posterior as:

$\hat{\alpha}=\int_{0}^{\infty} \alpha \frac{\alpha^{-p-1} e\left[-\left(\frac{\beta-H(\alpha, X)}{\alpha}\right)\right]}{\Gamma(p)[\beta+H(\alpha, X)]_{-}^{-p}} d \alpha$

$\hat{\alpha}=\frac{\beta-(m+1) \sum_{i=1}^{n-1} \lg \left(1-\alpha x_{i}\right)-\gamma_{r} \lg \left(1-\alpha x_{n}\right)}{p}$

\section{Applications}

The following data represent the observed failure times in hours of ten Gexp. Components. The data were actually simulated from Gexp. Population with $\alpha=0.96$

0.259810 .310080 .391690 .453750 .64403

0.745580 .797180 .812920 .884310 .96049

Two criteria, were employed for comparison of the parameter estimation, the set of criteria as follows:

a) The mean absolute errors (MAE)

The MAE test is based on the mean absolute distance between the $F\left(x_{i}\right)$ and the estimated $\hat{F}\left(x_{i}\right)$ [13], the MAE is given by:

MAE $=\frac{1}{n} \sum_{i=1}^{n}\left|\frac{F\left(x_{i}\right)-\hat{F}\left(x_{i}\right)}{F\left(x_{i}\right)}\right|$

b) The Root Mean Squares Error (RMSE) Statistic Test

The RMSE test is based on the mean squared distance between the observed $F\left(x_{i}\right)$

And the estimated $\hat{F}\left(x_{i}\right)$, [14], the RMSE is given by:

$R M S E=\sqrt{\frac{1}{n} \sum_{i=1}^{n}\left(\frac{F\left(x_{i}\right)-\hat{F}\left(x_{i}\right)}{F\left(x_{i}\right)}\right)^{2}}$

The smallest value of the test statistics in (a) and (b) is the better and indicates that data fits the chosen distribution.

Table 1: MLE of CDF, Survival and Hazard Rates for N=10, $\hat{\alpha}_{M L}=0.9046578, \alpha=0.96$

\begin{tabular}{lllll}
\hline $\mathrm{i}$ & $\mathrm{F} 0$ & FE & $\hat{R}(t)$ & $\hat{H}(t)$ \\
\hline 1 & 0.041 & 0.104 & 0.959 & 1.152 \\
2 & 0.051 & 0.114 & 0.949 & 1.164 \\
3 & 0.324 & 0.387 & 0.676 & 1.635 \\
4 & 0.420 & 0.483 & 0.580 & 1.905 \\
5 & 0.168 & 0.231 & 0.832 & 1.328 \\
6 & 0.115 & 0.178 & 0.885 & 1.249 \\
7 & 0.571 & 0.634 & 0.429 & 2.576 \\
8 & 0.740 & 0.803 & 0.260 & 4.250 \\
9 & 0.571 & 0.634 & 0.429 & 2.576 \\
10 & 0.176 & 0.239 & 0.824 & 1.341 \\
\hline
\end{tabular}


Table 2: Bayes Estimation of CDF, Survival and Hazard Rate Functions when $\mathrm{N}=10, \mathrm{~B}=2.6, \mathrm{P}=1, \mathrm{AB}=0.933$

\begin{tabular}{lllll}
\hline $\mathrm{i}$ & $\mathrm{F} 0$ & $\mathrm{FE}$ & $\hat{R}(t)$ & $\hat{H}(t)$ \\
\hline 1 & 0.041 & 0.074 & 0.926 & 1.158 \\
2 & 0.051 & 0.084 & 0.916 & 1.170 \\
3 & 0.324 & 0.357 & 0.643 & 1.667 \\
4 & 0.420 & 0.453 & 0.547 & 1.960 \\
5 & 0.168 & 0.201 & 0.799 & 1.342 \\
6 & 0.115 & 0.148 & 0.852 & 1.258 \\
7 & 0.571 & 0.604 & 0.396 & 2.708 \\
8 & 0.740 & 0.773 & 0.227 & 2.726 \\
9 & 0.571 & 0.604 & 0.396 & 1.355 \\
10 & 0.176 & 0.209 & 0.791 & \\
\hline
\end{tabular}

Table 3: Bayes Estimation of CDF, Survival and Hazard Rate Functions when $\mathrm{N}=10, \mathrm{~B}=3, \mathrm{P}=1.4, \mathrm{AB}=0.952$

\begin{tabular}{lllll}
\hline $\mathrm{i}$ & $\mathrm{F} 0$ & $\mathrm{FE}$ & $\hat{R}(t)$ & $\hat{H}(t)$ \\
\hline 1 & 0.041 & 0.096 & 0.904 & 1.161 \\
2 & 0.051 & 0.106 & 0.894 & 1.174 \\
3 & 0.324 & 0.379 & 0.621 & 1.690 \\
4 & 0.420 & 0.475 & 0.525 & 1.999 \\
5 & 0.168 & 0.223 & 0.777 & 1.351 \\
6 & 0.115 & 0.170 & 0.830 & 1.265 \\
7 & 0.571 & 0.626 & 0.374 & 2.805 \\
8 & 0.740 & 0.795 & 0.205 & 2.114 \\
9 & 0.571 & 0.626 & 0.374 & 2.805 \\
10 & 0.176 & 0.231 & 0.769 & 1.365 \\
\hline
\end{tabular}

Table 4: Bayes Estimation of CDF, Survival and Hazard Rate Functions when $N=10, B=2.333, P=2.4, A B=0.972$

\begin{tabular}{lllll}
\hline $\mathrm{i}$ & $\mathrm{F} 0$ & $\mathrm{FE}$ & $\hat{R}(t)$ & $\hat{H}(t)$ \\
\hline 1 & 0.041 & 0.117 & 0.883 & 1.165 \\
2 & 0.051 & 0.127 & 0.873 & 1.179 \\
3 & 0.324 & 0.400 & 0.600 & 1.715 \\
4 & 0.420 & 0.496 & 0.504 & 2.042 \\
5 & 0.168 & 0.244 & 0.756 & 1.361 \\
6 & 0.115 & 0.191 & 0.809 & 1.272 \\
7 & 0.571 & 0.647 & 0.353 & 2.916 \\
8 & 0.740 & 0.816 & 0.184 & 5.597 \\
9 & 0.571 & 0.647 & 0.353 & 2.916 \\
10 & 0.176 & 0.252 & 0.748 & 1.376 \\
\hline
\end{tabular}

Table 5: MAE and RMSE of MLE and Bayes Estimation Methods for N=10

\begin{tabular}{lllll}
\hline \multirow{2}{*}{ Test } & \multirow{2}{*}{ MLE } & \multicolumn{3}{c}{ Bayes } \\
\cline { 3 - 5 } & & $\mathrm{B}=2.333, \mathrm{p}=2.4, \alpha=0.972$ & $\mathrm{~B}=2.6, \mathrm{p}=1, \alpha=0.933$ & $\mathrm{~B}=3, \mathrm{p}=1.4, \alpha=0.952$ \\
\hline MAE & 0.026 & 0.053 & 0.125 & 0.036 \\
RMSE & 0.033 & 0.065 & 0.152 & 0.044 \\
\hline
\end{tabular}

\section{Remarks}

1) The maximum likelihood estimates of cdf, survival function and hazard rate functions are given in table (1), Bayesian estimates of cdf, survival function and hazard rate functions for different values of $B$ and $p$ are given in tables (2), (3) and (4).

The values of MAE and RMSE of maximum likelihood and Bayesian methods are given in table (5); the best method is the method that gives the smallest value of MAE and RMSE.

2) In table (1) when we computed parametric estimators of Gexp. Distribution in maximum likelihood (MLE) and Bayes using different values of B and p, we find from table (5) that the best estimator is the maximum likelihood method. 


\section{Conclusion}

The estimated parameter of Gexp. Distribution obtained from the maximum likelihood estimation is the best compared to Bayes estimation.

\section{References}

[1] Kamps, U. (1995 a), A concept of generalized order statistics. B. G. Teubner, Stuttgart. http://dx.doi.org/10.1007/978-3-663-09196-7.

[2] Ahsanullah, M. (1997), Generalized order statistics from power function distribution. J. of Appl. Statist. Sci., 5, 283-290.

[3] Ahsanullah, M. (2000), Generalized order statistics from exponential distribution. J. of Statist. Plann. And Inference, 79, 79-91. http://dx.doi.org/10.1016/s0378-3758(99)00068-3.

[4] Saran, J. and Pushkarna, N. (2000), Relationships for moments of order statistics from a generalized exponential distribution. Statistica, LX, No. 3, 585-595.

[5] Malinowska, I., Pawlas, P. and Szynal, D. (2006), Estimation of location and scale parameters for the Burr XII distribution using generalized order statistics. Linear Algebra and its Applications, 416, 150-162. http://dx.doi.org/10.1016/j.laa.2006.02.007.

[6] Al Omari,M. A., Hadeel, S. A. and Noor, A. I. (2010), Comparison of the Bayesian and maximum likelihood estimation for Weibull distribution. J. of Mathematics and Statistics, 6, 2, 100-104. http://dx.doi.org/10.3844/jmssp.2010.100.104.

[7] Abu El Fotouh,S. and Nassar, M. M.A.(2011). Estimation for parameters of the Weibull Extension model based on generalized order statistics. Int. J. Contemp. Math. Sciences, Vol. 6, no. 36, 1749-1760.

[8] Naser Odat (2010). Estimation of reliability based on Pareto distribution. Appl. Math. Sci. Val. 4, No. 55, $2743-2748$.

[9] Aljouharah, A. (2013). Estimating the parameters of an exponentiated inverted Weibull distribution under type-II censoring. Appl. Math. Sci., vol. 7, No. 35, 1721-1736.

[10] Attia, A. F., Shaban, A. S. and Abd El Sattar, M. H. (2013). Estimation in constant -stress accelerated life testing for birnbanum-saunders dietribution under censoring. Int. J. Contemp. Math. Sciences, Vol. 8, No.4, 173-188.

[11] Muhammad, Y. D. and Muhammad, A. (2013), Bayesian analysis of randomly censored generalized exponential distribution. Austrian J. of Statistics, Vol. 42, No. 1 47-62.

[12] Kamps, U. (1995 b), A concept of generalized order statistics. J. of Statist. Plann. And Inference, 48, 1-23. http://dx.doi.org/10.1016/03783758(94)00147-N.

[13] Mazen, Z. and Ammar, M. S. (2009), Parameters estimation of the modified Weibull distribution. Appl. Math. Sci., vol. 2, 11, 541-550.

[14] Singh, V. P. and Guo, H. (1995), Parameter estimation for 3-parameter generalized Pareto distribution by the principle of maximum entropy

[15] (POME). Hydrol. J. Sci., 40, 2, 165-181. http://dx.doi.org/10.1080/02626669509491402. 